\title{
Flujos, contrabando y prácticas de ilegalidad en la frontera México-Estados Unidos: cruces fronterizos entre Tijuana y San Diego
}

\section{Smuggling, illegality and circulation in the US-Mexico border: the case of Tijuana-San Diego}

Alberto Hernández Hernández ${ }^{\text {a* }}$ (i) https://orcid.org/0000-0001-7176-0902
Recibido el 2 de marzo de 2021.

Aceptado el 31 de agosto de 2021.

Publicado el 15 de septiembre de 2021.

${ }^{*}$ Autor para correspondencia: Alberto Hernández Hernández. Correo electrónico: ahdez@colef.mx

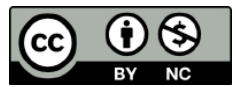

Esta obra está protegida bajo una Licencia Creative Commons Atribución-NoComercial 4.0 Internacional. a El Colegio de la Frontera Norte, Departamento de Estudios en Administración Pública, Tijuana,
México, correo electrónico: ahdez@colef.mx

\section{Resumen}

Este artículo describe los tipos de mercancías y objetos que cruzan la frontera México-Estados Unidos en ambas direcciones, particularmente el caso de las ciudades fronterizas de Tijuana-San Diego. Con aportes desde los estudios fronterizos se argumenta que la línea que divide a ambos países funciona de manera diferente de acuerdo con quién cruza y qué tipo de mercancías transporta. Al tomar esto en cuenta, por medio de datos etnográficos obtenidos durante las últimas dos décadas, el artículo analiza los tipos de mercancías legales e ilegales que circulan a través de la frontera. La diferencia entre el tráfico de mercancías y productos contrabandeados en dirección norte-sur y sur-norte permite no solo constatar los tipos de flujos transfronterizos, sino también inferir las necesidades y contextos de las personas que cruzan la frontera.

Palabras clave: Frontera México-Estados Unidos, Tijuana-San Diego, legalidad-ilegalidad, drogas, contrabando, armas de fuego.

\section{Abstract}

This paper explains the state of the United States-Mexico border regarding the smuggling of different types of merchandise, particularly in the Tijuana-San Diego border. It argues that the divide between both countries works differently depending on the direction and the type of merchandise being transported. Using ethnographic data collected during a 20-year period, the paper shows specific patterns of consumption that vary, depending on the direction in which the border is crossed. These differences go on to show the different wants and needs of people living in the border region.

Keywords: us-Mexico border, Tijuana-San Diego, legality-illegality, narcotics, smuggling, firearms.

CÓMO CITAR: Hernández, A. (2021). Flujos, contrabando y prácticas de ilegalidad en la frontera México-Estados Unidos: cruces fronterizos entre Tijuana y San Diego [Smuggling, illegality and circulation in the us-Mexico border: the case of Tijuana-San Diego]. Estudios Fronterizos, 22, e077. https://doi.org/10.21670/ref.2114077 


\section{Introducción}

Al tomar como caso de estudio la frontera México-Estados Unidos y de manera singular el trabajo de campo en la zona fronteriza entre Tijuana, Baja California, y San Diego, California, este artículo retoma las experiencias de cruce y contrabando de mercancías a través de la frontera para describir el tipo de objetos que suelen atravesar esta línea en ambas direcciones; con base en ello se indagan algunas de las dimensiones y prácticas que conviven día a día entre la legalidad y la ilegalidad, y que permiten pensar en las relaciones y tensiones en esta frontera. Lo anterior se sustenta en el trabajo de campo en estas ciudades fronterizas, investigación documental, así como testimonios y experiencias de informantes insertos en las dinámicas de contrabando e ilegalidad en esta frontera.

A partir de los límites internacionales fijados en 1846 entre ambos países, resultado de la guerra de intervención de Estados Unidos de América (EuA) contra México, la frontera que comparten ambos países tiene una extensión que abarca desde el océano Pacífico en Playas de Tijuana, al golfo de México en el estado de Tamaulipas. El Río Bravo o Río Grande ha servido como una barrera natural divisoria en la región noreste de México, que hace visible su cauce desde Ciudad Juárez, Chihuahua, hasta el golfo de México. Por otro lado, la región noroeste de esta frontera fue fijada con la instalación de obeliscos o mojoneras, además de basarse en divisiones geográficas establecidas por cerros, desiertos, esteros y cañones (Piñera, 1985).

Desde una visión panorámica, a la frontera norte mexicana se le ha dividido en las dos regiones referidas: noreste y noroeste, aunque, dependiendo de la literatura que se revise, también se puede hablar de una región central; en este artículo se parte de que existen las dos primeras. La noreste comprende a los estados de Chihuahua, Coahuila, Nuevo León y Tamaulipas del lado mexicano y por el lado estadounidense Texas y Nuevo México. En tanto que la región noroeste se encuentra integrada por los estados de Baja California y Sonora, que tienen como vecinos en el norte a California y Arizona en la Unión Americana (Figura 1). En conjunto "la zona fronteriza incluye 48 condados estadounidenses que se distribuyen en cuatro estados y 94 municipios mexicanos distribuidos en seis estados" (Senado de la República/Centro de Estudios Internacionales Gilberto Bosques, 2017). Para 2016, esta gran frontera en conjunto sumaba una población aproximada de 13000000 de habitantes distribuidos en 10 áreas metropolitanas transfronterizas (Prieto, 2016). ${ }^{1}$

Una de las principales diferencias en la frontera de México respecto a otras fronteras en América Latina es ser el punto de contraste que diferencia, y al mismo tiempo conecta, lo latino y lo anglosajón. La actual franja fronteriza entre México y Estados Unidos consiste en un territorio común que ha sido permeable en diferentes formas y momentos, además de servir como un mecanismo de filtración y de control entre dos estilos de vida separados no solo por el idioma y lo económico, sino también por lo histórico y lo religioso.

\footnotetext{
${ }^{1}$ Las diez zonas metropolitanas transfronterizas por orden de importancia en términos poblacionales son: Tijuana-San Diego; Ciudad Juárez-El Paso; Reynosa-McAllen; Matamoros-Brownsville; Mexicali-Caléxico; Nuevo Laredo-Laredo; Ciudad Acuña-Del Río; Nogales-Nogales; Piedras Negras-Eagle Pass; Agua Prieta-Douglas (Prieto, 2016).
} 
Figura 1. Zonas metropolitanas fronterizas entre México y Estados Unidos

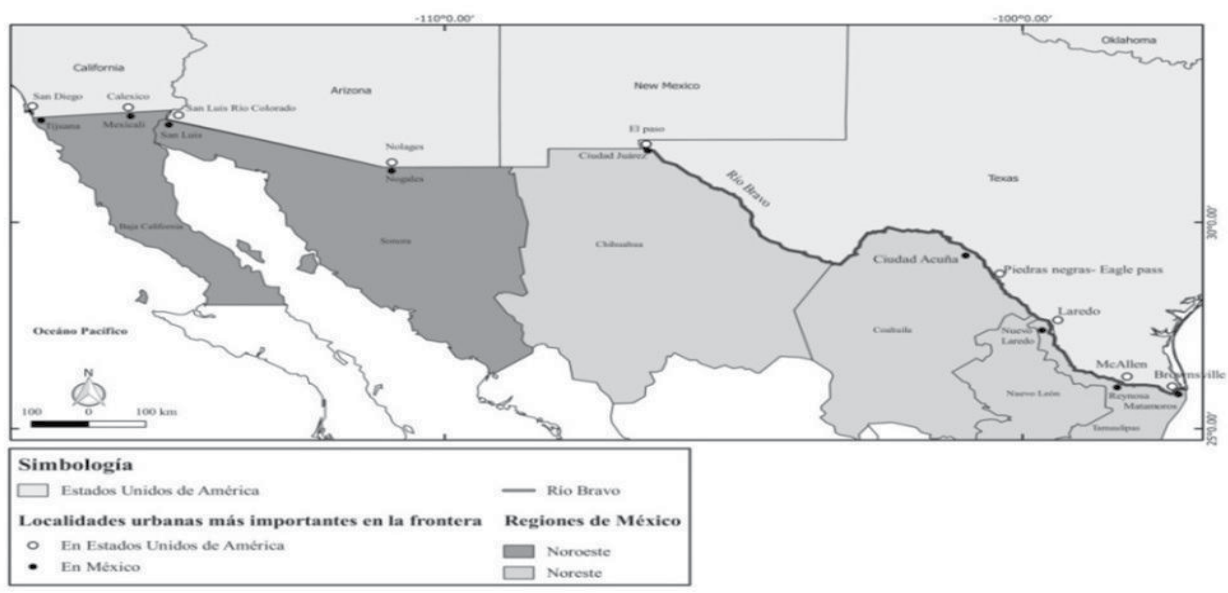

Fuente: Elaborado por Yasmin Ochoa, 2018

Millones de personas desarrollan sus vidas a lo largo y ancho de esta franja fronteriza. La disparidad que se puede observar entre estos países, así como los contrastes entre la oportunidad y la carencia, refuerzan aún más lo singular de la llamada frontera más transitada del mundo. Filtros y control expresan dos ejes de la vida, aunque a la par de estos podrían añadirse los ejes del beneficio y la oportunidad, sobre todo al hablar de lo considerado legal e ilegal en cada país.

Pese al incremento de filtros, controles, barreras y muros en las últimas cuatro administraciones estadounidenses, esta zona fronteriza ha configurado sus propias dinámicas de interacción y contacto. Testimonio de ello es la alta cantidad de cruces fronterizos registrados a lo largo de las últimas cuatro décadas. De acuerdo con datos del Bureau of Transportation Statistics (втs), en 2019, de México hacia Estados Unidos, se registraron cerca de 50000000 de cruces peatonales, poco más de 212000000 de cruces vehiculares y casi 12000000 de cruces de camiones y vehículos de carga a través de 55 puertos fronterizos en la frontera. Destaca el puerto de San Ysidro con 10799398 de cruces peatonales y 40942771 de cruces vehiculares. En tanto, Laredo sobresale por ser el puerto más activo en materia de camiones y contenedores de carga, con más de 4345344 cruces en 2019. Si se toman en cuenta estos flujos en sentido inverso, la cifra aumentaría considerablemente (BTs, 2019).

Si bien, hay un flujo transfronterizo continuo entre los dos países, otra de las distinciones a considerar es que la vecindad de México con Estados Unidos posee acentuadas diferencias según la región donde se ubique. Esto, en el sentido de que no es lo mismo pensar en las interacciones entre Baja California y California, por ejemplo, frente a las interacciones entre Sonora y Arizona, o Tamaulipas con Texas. Aunque estos sectores forman parte de la misma región fronteriza, los grados de interacción económica, social y cultural son diferentes en su composición y características, prueba de esto es la intensa actividad económica, social y cultural entre Baja California y California, al ser este último uno de los principales motores económicos en Estados Unidos, además de concentrar una alta cantidad de habitantes de origen mexicano en su territorio. 
El artículo se estructura de la siguiente manera: en una primera instancia se explica brevemente el proceder metodológico, para luego, desde la academia, dar entrada a una revisión sobre la ilegalidad y el contrabando; en este mismo sentido se aborda una reflexión sobre los mercados ilegales transfronterizos entre México y Estados Unidos, lo cual sirve como antesala para hablar sobre el caso particular de la frontera entre Tijuana y San Diego y las experiencias en torno al contrabando cotidiano, así como casos de tráfico de armas y túneles transfronterizos.

\section{Metodología}

La información etnográfica que da soporte a las afirmaciones que se hacen en este artículo fue obtenida mediante los siguientes métodos: observación participante, observación directa y entrevistas a profundidad. Este proceso se vio potenciado gracias a una serie de contactos establecidos durante la trayectoria dentro de diferentes círculos de contrabando de ropa, medicamentos, productos y equipo de construcción, así como artículos para el hogar. Esta experiencia en particular permitió llevar a cabo entrevistas a dichas personas, entre las cuales pueden encontrarse pequeños comerciantes, choferes, propietarios de farmacias e individuos cuyo cruce regular les permitía circular mercancías en ambas direcciones. Esta aproximación facilitó que aparecieran relatos presentes en el imaginario de quienes cruzan mercancía de contrabando, de manera cotidiana, en esta parte de la frontera.

Además de ello, la experiencia de recorrer, a lo largo de varias décadas, las principales ciudades de la frontera norte ha permitido conocer y experimentar el fenómeno migratorio de manera directa, así como conocer parte de la vida cotidiana en dichos lugares. Aunado a ello, la experiencia de trabajar con un estudiante que desarrolló su tesis de doctorado en El Colef sobre el consumo transfronterizo de cannabis, facilitó la expansión de las redes de contactos, lo cual permitió que se considerara ese ámbito del contrabando. Es a través de esta combinación de contactos y experiencia laboral que se pudo entrevistar a individuos clave relacionados con la actividad de contrabando en la región Tijuana-San Diego. Es importante mencionar que el propósito de las entrevistas realizadas fue el de construir una descripción de los puntos más relevantes en la región en lo que refiere al contrabando de mercancías; en ese sentido, este artículo hace énfasis sobre las narrativas relacionadas a esta actividad y no necesariamente sobre las experiencias personales de los actores involucrados.

\section{llegalidad y contrabando}

Los estudios sobre fronteras han transitado en temas y enfoques que se corresponden con sus propias realidades socio-espaciales. Esto se distingue en el continente americano y en la composición de sus diferentes regiones. Si antes los estudios sobre fronteras se referían a la comprensión geográfica y territorial (Brigham, 1919), ciertas tipologías de las fronteras (Hartshorne, 1936) y su anclaje con las delimitaciones de los Estados-nación (Donnan \& Wilson, 2001), posteriormente habría un énfasis en las interacciones sociales y culturales que se configuran en estos espacios (Anderson 
\& O’Dowd, 1999; Newman, 2006), así pueden observarse distintas propuestas de tipologías para el estudio de las regiones transfronterizas (Dilla Alfonso \& Neira Orjuela, 2020), cada uno de estos estudios aportaron una mirada cualitativa, multidimensional y multiescalar sobre fronteras y regiones transfronterizas, lo que permitió indagar más en torno a las subjetividades, prácticas y relaciones de poder.

Ante una comprensión de las fronteras que se actualiza por fenómenos y condiciones situadas, su estudio demanda no solo identificar características territoriales, económicas y poblacionales, sino aquellas relaciones de poder que transforman la vida en estos espacios, algunas de ellas son las prácticas entre lo legal y lo ilegal. Por estos términos se entiende que lo legal/ilegal está circunscrito por la definición que el Estado haga sobre ello, según las normatividades que los principios jurídicos establezcan, y que esta división cambiará en función del contexto espacial y temporal, tal como lo señala la geógrafa brasileña Lia Osório Machado (2000): una necesaria distinción al pensar las fronteras y su relación con lo legal y lo ilegal es diferenciar las nociones de límite, asumido como "un instrumento de separación entre unidades políticas soberanas" (Osório Machado, 2000, p. 9) y donde los circuitos de la ilegalidad contribuyen a definir lo que está permitido o no, según el lugar donde se establezca tal distinción. Otra de las diferencias que subraya Osório es la comprensión del territorio, misma que cambia a partir de la proliferación de leyes, normas y reglas en el interior de los territorios nacionales; reglamentaciones y criterios válidos para ciertos sectores y lugares, pero no para otros. Nociones que Osório identifica como "territorios-objeto", en tanto que están diseñados para atender objetivos específicos que sirven como criterio legal para acciones consideradas como ilegales en el país de origen, por ejemplo, paraísos fiscales, centros financieros offshore, entre otros (Osório Machado, 2000, p. 11). Estos procesos señalan un cambio de paradigma en cuanto a la comprensión del territorio y límite en los espacios fronterizos, especialmente frente a lo que se designa como legal e ilegal:

La historia de los pueblos y las instituciones muestra que la noción de legalidad/ilegalidad es intrínseca a toda organización social, pero la condición de legalidad o de ilegalidad de cualquier acción es mutable en el tiempo y el espacio. Esto ocurre porque las normas jurídicas, las circunstancias diplomáticas y los dispositivos técnicos movilizados generan concepciones de tiempo y espacio distintas. En esas circunstancias, las definiciones de tiempo y espacio son de corta duración y los eventos a ellas relacionados son provenientes del contexto inmediato [A história dos povos e das instituições mostra que se a noção de legalidade e ilegalidade é intrínseca a toda organização social, a condição legal ou ilegal de qualquer ação é mutável no tempo e no espaço. Isso ocorre porque as normas jurídicas, as circunstâncias diplomáticas, os dispositivos técnicos mobilizados geram concepções de tempo e espaço distintas. Aqui se pretende comparar dois momentos da história dos limites e fronteiras do Brasil. Embora sujeitos a condições bem diferentes de imposição do que era legal e ilegal, e a concepções distintas de tempo e espaço, têm em comum a curta-duração e a dependência em relação a eventos provenientes do contexto imediato]. (Osório Machado, 2000, p. 11) ${ }^{2}$

\footnotetext{
${ }^{2}$ Traducción propia de Osório Machado (2000, p. 11).
} 
Además de la condición situada para la comprensión de límite y territorio, así como las concepciones de espacio y tiempo por los límites jurídicos y la actuación institucional en fronteras, otra de las discusiones que propone Osório Machado, junto a Reyes y Monteiro (2009) es profundizar el concepto de "zonas fronterizas" a partir de tres condiciones: 1) los diferentes sentidos en torno a la integración regional; 2) la simbiosis entre las actividades económicas legales e ilegales; y, 3) la temporalidad de las respuestas a nivel local y regional, ante los cambios de elementos que reconfiguran la geografía económica en zonas de frontera.

Para Osório Machado y colaboradores (2009), el concepto de frontier (frontera) es más rico que otros como border, borderland o border regions, ya que, si bien, una frontera en su origen etimológico alude a un límite real o metafórico, también indica el inicio de algo diferente (Osório Machado et al., 2009, p. 98). Por ello, el concepto de frontier permite dar cuenta de espacios en constante interacción pese a la demarcación internacional, ya que este vocablo se liga más a su sentido de cruzar límites, en oposición a otros conceptos más categóricos al enfatizar la demarcación como border, por ejemplo. Las zonas fronterizas se distinguen por las diversas formas de interacción que ocurren en ellas pese a los límites estatales que separan el territorio, en este sentido, hablar de zona fronteriza es un concepto pertinente que ayuda a plantear las relaciones entre lo legal y lo ilegal que acontecen en espacios como el de la frontera de México y Estados Unidos.

Esto no quiere decir que las asimetrías entre ambos países se disuelvan a través de los contactos y las interacciones en la frontera, al caer en las visiones esencialistas que advierte Grimson (2011) sobre los sentidos de la "hermandad" o la "hibridación generalizada". Esencialismos en los que se desdibuja el papel de los Estados en la vida de las personas que viven en fronteras (Grimson, 2011, p. 114). Más bien, la propuesta de Osório Machado y colaboradores (2009), aun cuando son estudios centrados en fronteras sudamericanas, contextualizan cómo son reconfiguradas las zonas de frontera a través de flujos dinámicos, sin perder de vista que las poblaciones fronterizas reivindican sus referentes de identidad, así como elementos y símbolos culturales específicos, tal y como ocurre en la zona fronteriza mexicoestadounidense.

Para el caso específico de la zona fronteriza Tijuana-San Diego en Baja California y California, respectivamente, la cotidianidad transcurre entre el orden y lo incierto, el control y lo clandestino. Aunque ciertos flujos legales son identificables gracias a los dispositivos de registro y la infraestructura de vigilancia y seguridad que administra el cruce de personas y mercancías, la transgresión ilegal es una condición que está presente en el día a día de los puertos fronterizos. La relación bilateral entre México y Estados Unidos se caracteriza por el énfasis particular en el combate a la introducción de productos y mercancías que, por razones de seguridad, sanitarias y arancelarias, se encuentran restringidas; un ejemplo de este tipo de restricciones son las armas, las drogas, los medicamentos controlados, cigarros, alcohol, ciertos tipos de alimentos, ropa usada y material de construcción, entre otros. Para el caso de esta frontera, la Oficina de Aduanas y Protección Fronteriza (СBP por sus siglas en inglés) de Estados Unidos, en conjunto con la Administración General de Aduanas de México, con base en las normativas de diversas agencias y secretarías gubernamentales, determinan con detalle el tipo de productos permitidos y prohibidos. Además de ello, se debe tener presente que "La frontera no solo es la 'línea de las aduanas' (Grimson, 2003, p. 14; Kearney, 2006, p. 32) sino también un elemento para estudiar las estructuras simbólicas, las relaciones de poder y las cuestiones ideológicas propias de las sociedades (Heyman, 1994, p. 58)" (Sandoval, 2012, p. 16). 
La acción de transgredir esta zona de frontera se podría identificar en al menos dos sentidos: el primero, como experiencia corporal, en tanto que la transgresión implica distintos efectos para quien la identifica y la contiene, como para quien la planea y la realiza. Y el segundo sentido, como experiencia del movimiento, ya que la transgresión adquiere matices distintos si el cruce se realiza de sur a norte, de norte a sur, o en cualquier dirección que traspase límites internacionales.

De modo que en zonas fronterizas como la de Tijuana y San Diego, los sentidos de transgresión se superponen y confunden, pues para quien resguarda el límite fronterizo de sur a norte es un deber y una misión evitar la transgresión, pero para quien busca atravesar la frontera de sur a norte es un desafío por superar sea legal o ilegalmente.

Pensar la transgresión en referencia al cruce de norte a sur conlleva otro conjunto de efectos y aspiraciones, ya que para la población que no es de Estados Unidos, el cruce de frontera implica ajustarse a un orden y a un conjunto de reglas del lado estadounidense; por el contrario, para quien realiza el cruce de euA hacia México, en ocasiones, busca alejarse de esas mismas reglas en el país vecino.

Se trata entonces de tensionar argumentos que solo ven el tráfico y el contrabando de modo unidireccional, por ejemplo, de sur a norte, cuando estas prácticas ocurren en ambas direcciones. Y donde el conjunto de motivaciones y efectos que generan tienen impactos diferenciados en la vida cotidiana de una frontera. Un estudio de caso que ilustra estas dinámicas fue realizado por Sandoval en la frontera de Texas con Tamaulipas, donde se analiza el control territorial del comercio de ropa usada en dicha región (Sandoval, 2018).

Es importante no olvidar que "si no existiera una frontera, dos sistemas de producción distintos y dos mercados separados, no habría ninguna razón para contrabandear" (Dorfman, 2020, p. 155). Tampoco se debe imaginar al contrabandista como una persona estigmatizada y fuera de la ley, el contrabandista es un sujeto que transita entre lo legal y lo ilegal, por lo que el contrabando no se posiciona fuera de la economía legal, sino que es parte de ella, como una economía gris. Este término, sin embargo, solo aplica para aquellos productos que son parte del mercado legal; armas y drogas rara vez entran en el mercado legal y deben recurrir a otras estrategias para su tráfico (Dorfman, 2020).

\section{Mercados informales transfronterizos}

Los grandes mercados clandestinos dedicados al tráfico de drogas y de personas (que por su complejidad y características son temas que no se profundizan en este trabajo) son circuitos de la ilegalidad con una presencia de décadas en la región. Un estudio publicado por Fuentes (2017) aborda la existencia de nodos del sistema global de las drogas prohibidas, y que para el caso mexicano, las fronteras terrestres, marítimas y aéreas de México, tienen un papel de nodos del flujo de los mercados ilícitos en esta red global, que provocan graves secuelas en el ámbito de la seguridad, el incremento en el número de homicidios dolosos y la descomposición social que generan estos fenómenos en los lugares donde se presentan (Fuentes, 2017). Este estudio destaca el caso particular de la región fronteriza con Estados Unidos, en tanto que los principales corredores y rutas de las drogas ilícitas atraviesan las regiones noroeste, centro y noreste de México, en busca de llegar al país de destino en el norte. 
En lo que respecta al tráfico de personas por la frontera norte de México, a partir de datos obtenidos en trabajo de campo, se destaca el hecho de que, durante las décadas de 1980 y 1990 e inicios del siguiente milenio, el cruce clandestino de personas a Estados Unidos era operado por diferentes actores e incluso por redes familiares, conocidos localmente como coyotes o polleros. Sin embargo, desde 2006 y hasta la fecha, el tráfico de personas mayoritariamente lo realizan integrantes del crimen organizado ligados a los cárteles del narcotráfico, quienes no solo encarecieron el cobro por el cruce clandestino según nacionalidad y edad, sino que hicieron un negocio redituable al utilizar la extorsión, el secuestro y abusos a migrantes como vías para allegarse recursos.

Actualmente se contempla el fenómeno de nuevas movilidades como las caravanas de migrantes, para quienes la vía clandestina ya no era una opción por la cantidad de riesgos a los que se exponían, y recurrieron a movilizarse a través del acompañamiento de asociaciones civiles y medios de comunicación, como alternativas para protegerse en su camino por México (Hernández, 2018).

Además de estos grandes mercados informales, en el que también se situaría el tráfico de armas de norte a sur, y que por su complejidad son los más visibles y urgentes de atender en términos de seguridad, se encuentran también aquellos mercados informales cuyos efectos e impactos son más de orden económico y social en las zonas fronterizas.

Durante los últimos 20 años, la seguridad fronteriza se ha incrementado de manera importante a raíz de los ataques terroristas de 2001. A pesar de este incremento en la seguridad en los puertos fronterizos, las personas siguen contrabandeando productos prohibidos como alimentos perecederos, café, pan, aceite de cocina, productos enlatados, dulces, salsas, entre otros. La información de los párrafos siguientes es producto de observación directa, observación participante y también de los relatos expuestos por las personas entrevistadas; de ninguna manera pretenden reflejar cantidades de corte estadístico o presentarse como muestras representativas; estas condiciones no le restan relevancia a las narrativas expuestas; aun así, existen algunos casos en los que sí es posible encontrar una referencia estadística cuantitativa, como los reportes de la BTs y de la Oficina de Fiscalización General de Estados Unidos.

Aunque las personas que contrabandean se exponen a recibir castigos sobre sus privilegios de cruce, o ser acreedoras a multas importantes, es común que suelan protegerse diciendo que están cruzando mandado. Luego de las preguntas hechas por los oficiales de la garita, las personas deben pasar sus bolsas y maletas por una máquina de rayos $\mathrm{X}$ para una revisión posterior, aunque en este punto, el castigo no implica procedimientos mayores, pues de ser descubiertos, la consecuencia será la remoción de los artículos prohibidos antes de su acceso a Estados Unidos. Depende del tipo de producto contrabandeado y las respuestas dadas durante el cuestionamiento, las personas pueden ser enviadas a una revisión secundaria, aunque la clave en algunos casos para superar este tipo de interrogatorio suele ser el decir que las bolsas sospechosas contenían sus almuerzos.

Entre los traficantes hormiga, hay especialistas en productos particulares, tales como medicinas, productos naturistas, productos homeopáticos y hierbas curativas (Loya et al., 2009). La mayoría de las ciudades mexicanas tienen mercados destinados a la venta de estos productos, y ha sido una práctica relativamente común que las personas de origen hispano crucen a México para adquirir hierbas difíciles de encontrar en Estados Unidos. Para el caso de Tijuana, el centro urbano, por ejemplo, contiene decenas de farmacias y tiendas naturistas. En una de las entrevistas que se llevó a cabo con el 
dueño de una farmacia en Tijuana se mencionaron dos personajes interesantes: el primero, un viejo médico egresado de la Universidad Nacional Autónoma de México con residencia en Los Ángeles, el cual obtenía medicamento en México, con el fin de venderlo. Para disminuir el riesgo de ser descubierto en la garita, el personaje indicaba al dueño de la farmacia que se deshiciera de los empaques de las medicinas. Una vez hecho esto, mezclaba todo el producto con dulces y chocolates, y pan dulce en una bolsa; así al cruzar la garita si el oficial le preguntaba “¿Hay algo que declarar? él podría indicar "solo dulces mexicanos y pan dulce".

Otro individuo era un estadounidense rubio, delgado, de unos 50 años, conocido como el meón, el cual siempre solicitaba usar el baño de la farmacia con el fin de amarrar los medicamentos a sus piernas para emprender el cruce de vuelta a Estados Unidos. Ese cliente, compraba medicamentos controlados que solo se adquieren con receta médica. La dinámica era: "despistar las miradas", en donde los dueños de la farmacia solían regalarle medicamentos como aspirinas y analgésicos, y productos como sodas, jugos, botanas, dulces, souvenirs, y hasta un recibo falso le emitían. Así, si la policía lo abordaba, él podría decir "solo compré analgésicos y unos snacks". En aquel tiempo ya era usual que policías municipales se dedicaran a intimidar y extorsionar a turistas estadounidenses, los amedrentaban diciendo que lo que habían comprado en esas farmacias era "ilegal" y si no les pagaban una "cuota" podían llevarlos detenidos. Nunca comentó su nombre real, no tenía un horario o día definido para hacer sus compras. Su vestimenta siempre era casual-formal, como si trabajara en una oficina. Los dueños de las farmacias suponían que eso era parte de sus estrategias para no levantar sospechas. De esa manera, si al cruzar la frontera el oficial свP le preguntaba “¿Algo que declarar?” él diría "nada, solo dulces y aspirinas".

El tráfico hormiga tiene características diferentes según el tipo de flujo que se analice: mientras que en dirección sur suele girar en torno a armas de fuego legalmente obtenidas, ropa usada, alimentos y dispositivos electrónicos, el flujo en dirección norte consiste generalmente en medicinas, productos herbolarios y homeopáticos y "productos de nostalgia", destinados a un mercado de origen hispano que tiene pocas oportunidades de regresar a sus regiones y países de origen. A pesar de esto, las formas de llevar a cabo este tipo de prácticas, sobre todo de México a Estados Unidos, han sido poco estudiadas y la información referente a este fenómeno suele centrarse en el tráfico de sustancias ilícitas a través de vehículos y personas, por lo que generalmente se conoce a través de notas periodísticas.

Así, el contrabando de ropa, muebles, artículos electrodomésticos, medicamentos, alimentos no perecederos y automóviles, entre otros, ha sido aprovechado durante décadas por las poblaciones fronterizas, al adquirir esos productos a un precio considerablemente menor al que tienen en el resto de México. Una de las estrategias para las personas que compran grandes cantidades de mercancías es, si son enviados a revisión secundaria, cruzar con varios miembros de la familia en el mismo auto para dividir las compras y así no sobrepasar el límite permitido por persona, que es de 300 usD y en época navideña 500 USD. Una segunda estrategia para evitar pagar impuestos o el decomiso de los artículos es "dividir" la mercancía o los artículos en varios cruces para evitar hacer declaraciones y pagar impuestos ante el Servicio de Administración Tributaria (SAT). Además, algunas personas comentan que una práctica común es que si compran computadoras, artículos eléctricos medianos o cantidades moderadas de ropa en San Diego, antes de cruzar la garita les retiran empaques y etiquetas, por si son enviados a revisión, puedan indicar ante los agentes del sat que el artículo no es nuevo. 
Por otro lado, de manera general se suele pensar que la población en la frontera norte de México es la principal beneficiaria del contrabando de mercancías, productos y demás objetos de desecho que provienen de Estados Unidos. Sin embargo, también la población estadounidense se beneficia de este mercado informal transfronterizo, al tener la posibilidad de deshacerse de mercancías, artículos y objetos, que si son almacenados representan un riesgo legal y un alto costo económico por las multas e infracciones que pudieran generar, sin mencionar también el impacto ambiental, así como otras sanciones por acumulamiento en los Estados Unidos. Ejemplo de esto se presenta en el condado de San Diego, California, donde la población no tiene permitido tener un cierto número de muebles o mobiliario de grandes dimensiones bajo resguardo en sus casas, ni tampoco pueden deshacerse de ellos en sitios abandonados o vías públicas, ya que se hacen acreedores a altas multas y antecedentes penales con las autoridades de ese país. Para estos casos se recurre a organizaciones que reciben este tipo de mercancías y artículos en calidad de donativos, o bien, aprovechan los circuitos del mercado informal transfronterizo para despojarse de lo que consideran deshecho.

Lo mismo ocurre con el caso de las pinturas acrílicas destinadas para la construcción de casas o edificios en Estados Unidos, donde la empresa contratista en Estados Unidos no puede deshacerse de la pintura excedente, ya que esta es considerada como desecho químico y requiere un tratamiento especial. Ante esta circunstancia tienen dos opciones: $a$ ) entregarla en calidad de donativo a asociaciones civiles y organizaciones de caridad; o, $b$ ) regresar el producto a la empresa proveedora, quien se encarga de administrar los excedentes de pintura, sin recibir por ello ninguna retribución.

Si se sigue la opción $b$, el excedente de pintura puede ser ingresado a México tras el pago del impuesto a la Aduana o cruzarlo mediante distintas modalidades de contrabando. En la ciudad de Tijuana hay locales comerciales dedicados a la venta exclusiva de pintura acrílica proveniente del vecino país. Algo visible al promocionarlas en México con el etiquetado de "pinturas americanas". Juan, un vendedor de pinturas que lleva más de 10 años dedicándose a esa actividad, tiene un pequeño puesto en un mercado sobre ruedas donde vende cubetas y galones de pintura a un bajo precio. Juan comenta que en su inventario llega a tener hasta 100 cubetas por semanas que va reemplazando según va vendiendo. El proveedor se las entrega directamente en su local. Otra experiencia de comercialización muy extendida es la venta de madera que antes pertenecía a casas no habitadas o en abandono en San Diego, las cuales pasan por un esquema similar de donación y rehúso en la frontera. En Tijuana, Palemon es el encargado de un local de venta de madera usada que llega de Estados Unidos. En ese lugar, cada semana descargan un tráiler de tablones, vigas, barrotes y puertas de garaje. En el proceso se desmontan piezas, y la madera se limpia para eliminar clavos y herrajes, para posteriormente ser vendida.

De modo que un esquema tentativo para referir las dinámicas legales e ilegales en zonas fronterizas como la de San Diego es definir qué mercancías o productos que son legales en Estados Unidos se tornan ilegales al aumentar en cantidad y volumen y no contar con un lugar especial para su almacenamiento. Por lo que una alternativa es cruzarlas al lado mexicano, donde son internadas por la vía legal, o bien, a modo de contrabando hormiga o en vehículos de transporte que requieren cubrir "cuotas especiales" (sobornos) a las autoridades aduanales y a la policía, según el tipo y la cantidad de mercancías que internan en el país.

Existe una diversidad de artículos o productos de desecho en el lado estadunidense que encuentran un nuevo uso y periodo de vida en el lado mexicano, lo que favorece 
en gran medida a personas de bajos recursos. Estos productos son vendidos en los mercados sobre ruedas, o mercados ambulantes, en los que miles de vendedores se encargan de comprar y distribuir a bajo precio productos, muebles y alimentos no perecederos que provienen de Estados Unidos. La señora Juanita se ha especializado en la venta de alimentos no perecederos y frutas. Su rutina consiste en instalarse de manera itinerante en cinco mercados ambulantes localizados en distintos puntos de la ciudad. Tan solo en Tijuana existen alrededor de 600 mercados sobre ruedas, distribuidos por las nueve delegaciones municipales. Cada día de la semana y de manera rotativa hay colonias específicas en las que se instalan estos mercados, los días de mayores ventas son los fines de semana (viernes, sábados y domingos). Algunos de los principales mercados sobre ruedas en Tijuana se ubican en la colonia Francisco Villa, el mercado del Mariano Matamoros y el mercado en La Morita. Una característica que destaca es que la mayoría de sobre ruedas en Tijuana se ubican hacia el lado este de la ciudad, en dirección al municipio de Tecate. En Tijuana, la mayoría de estos mercados sobre ruedas son matutinos con horarios de seis de la mañana a dos o tres de la tarde. Sin embargo, en otras ciudades fronterizas como Ciudad Juárez, estos mercados funcionan también por las noches, lo que se debe a los distintos horarios laborales de las empresas maquiladoras.

Otro de los aspectos a considerar son los actores involucrados en estos mercados, muchos de ellos agentes transfronterizos que acuden a ciudades como Los Ángeles o San Diego, California, para obtener mercancías y después transportarlas a la frontera con México. Desde una mirada etnográfica, el estudio de Sandoval (2012) es ilustrativo para mostrar cómo se articulan las redes sociales transfronterizas en esta dinámica de los mercados informales en la frontera noreste de México con Estados Unidos.

Otro conjunto de actores que participan en estas redes son agentes de la aduana y la policía, quienes forman parte de este tipo de economía a través del cobro de "cuotas" según el tipo y la cantidad de mercancías que se pretendan ingresar a territorio nacional. Una vez en México, la distribución sigue distintos canales, entre ellos están los mercados sobre ruedas y las tiendas de artículos de segunda mano. Algo característico de estas prácticas de contrabando es que los actores que se encargan de la compra, transporte, cruce y venta, en la mayoría de los casos son redes familiares transfronterizas. Como se mencionó, las restricciones impuestas sobre la frontera por las diversas agencias de Estados Unidos y de México adquieren características propias al territorio. En ese sentido, lo legal y lo ilegal se concibe y se negocia de maneras particulares que difieren con el resto de ambos países. Bajo la figura del contrabando, los diversos actores introducen mercancías de forma subrepticia que pueden superar la distancia institucionalizada en la frontera entre estos países, dando con ello un sentido amplio de movilidad a las personas y a los objetos; con esto último se hace referencia a los requisitos, muchas veces imposibles de cumplir para numerosas personas, que permiten un tránsito documentado y libre entre ambas naciones.

\section{Experiencias en torno al contrabando cotidiano en la frontera}

El cruce de fronteras es una oportunidad para pasar de un lugar a otro, observar contrastes, jugar un rol diferente en cada uno de los países, o incluso, una oportunidad para manejarse entre las posiciones difusas de la ilegalidad, en tanto que las zonas de frontera también se caracterizan por la permeabilidad y dinamismo que posibilitan. 
En un espacio como la frontera México-Estados Unidos, los sentidos del flujo de personas y mercancías, tanto de sur a norte como de norte a sur, adquieren grandes diferencias no solo en términos de magnitud, sino en la oportunidad de asumir que la ilegalidad tiene formas de ser puesta en práctica. La idea de que las fronteras son áreas sin ley quizá suena algo exagerado, pero existen prácticas y redes locales e internacionales en las que se acumulan recursos y se desarrollan habilidades para evadir disposiciones normativas legales; si bien este tipo de procesos no es exclusivo de las regiones fronterizas, la alta dinamicidad en términos de flujos de personas y mercancías hacen que su estudio sea particularmente relevante. La existencia en estos espacios de cierto tipo de mercados ilegales cobra fuerza y sentido, y dependiendo desde dónde se les vea, poseerán un significado distinto: para el Estado este tipo de prácticas son actos contra la ley; pero para algunos actores son oportunidades de allegarse recursos, aun cuando provengan del mercado informal.

La vida cotidiana en una frontera como la de México y Estados Unidos convive entre estos contrastes al aprovechar las oportunidades que otorga su condición fronteriza, pero sortea sus problemáticas y crisis por esta misma condición. Retos y oportunidades caracterizan la convivencia que mantienen estas dos sociedades. Para el caso de la frontera Tijuana-San Diego, hechos concretos que muestran las relaciones entre lo legal y lo ilegal pueden encontrarse, por ejemplo, el que la mayoría de edad en California es de 21 años y en Baja California de 18 años, lo que vuelve a Tijuana un lugar privilegiado para la vida nocturna de jóvenes estadounidenses desde hace décadas.

Otro ejemplo es el tema de la regulación del cannabis, en territorio californiano es legal su uso medicinal y recreativo, en tanto que, en el lado mexicano, su comercio sigue un esquema restringido de regulación y continúa siendo perseguida y castigada su venta y consumo. Un estudio más detallado sobre cambios en las políticas de regulación de cannabis en la frontera lo realizó Vinasco (2018), quien aborda las prácticas de consumo transfronterizas entre Tijuana y San Diego a partir de los distintos esquemas de regulación y control en cada Estado y país.

Un ejemplo adicional es en cuanto al uso y posesión de armas de fuego, incluidas armas de alto poder, permitida su venta y comercialización del lado estadounidense, y prohibidas en México. Resulta paradójico observar en las orillas del freeway de San Diego, y a unos pasos de la garita internacional, un anuncio oficial del gobierno estadounidense para viajeros que cruzan la frontera: "Medical marijuana prohibited into Mexico, prohibited into USA" y otro que advierte: "Guns are illegal in Mexico".

Aunque el establecimiento de sistemas de contrabando en torno a la ropa existe en otros puertos fronterizos, como es el caso de El Paso-Ciudad Juárez (Gauthier, 2009), el volumen y la intensidad con la que ocurre este tipo de tráfico en la frontera Tijuana-San Diego hace que se trate de un caso particular. El caso de la ropa y zapatos usados son casos interesantes, ya que gran parte de ellos son recolectados por tiendas de caridad como Salvation Army, Goodwill o por su venta en los llamados garage sales en distintos vecindarios californianos. Para dichas mercancías y productos está prohibido su ingreso a México por razones sanitarias, pero en Estados Unidos su comercialización es permitida. El trabajo de Efrén Sandoval sobre las "pacas de a kilo" en la frontera texana refleja un sistema de comercio particular de la zona, donde existen grandes bodegas en las que se vende ropa usada, que es trasladada a México a través de vehículos especializados, esta actividad transfronteriza se hace a través del personaje tradicional llamado "pasador" (Sandoval, 2018, p. 327); al parecer, cada ciudad fronteriza puede tener una dinámica en lo que se refiere a la comercialización y contrabando de ropa 
usada, tal y como podemos observar para el caso de Tijuana-San Diego. Mediante información reunida en trabajo de campo, informantes en Tijuana dedicados al contrabando de ropa usada declaran que existen diferentes formas de introducirla desde Estados Unidos. La forma en que familias o pequeños comerciantes cruzan estas mercancías a México es a través del llamado "contrabando hormiga", para ello usan pacas, bolsas o maletas para trasladarlas, o bien, emplean vehículos conocidos como "paneles", en donde se transportan mercancías y artículos en mayor cantidad.

De igual manera, como se ha compartido antes, en Estados Unidos hay otros artículos que están en una transición entre lo legal y lo ilegal, sea por su número, volumen o tipo de producto como muebles y colchones usados, electrodomésticos de desecho, llantas y vehículos, los cuales son trasladados al lado mexicano para su aprovechamiento y reciclaje. Un caso especial es el de los automóviles usados provenientes de Eu, llamados, en Tijuana y en todo México, "carros chocolate", automóviles con registro en Estados Unidos, pero sin registro del lado mexicano. Muchos de estos autos ya no pasan la verificación ambiental vehicular en EU, pero pueden circular en el lado fronterizo de México, lo que representa un desafío para las autoridades locales en términos de movilidad y seguridad urbana.

Otro caso que ilustra estos contrastes entre lo legal y lo ilegal ocurre con los medicamentos controlados, que son adquiridos con mayor facilidad en México que en Estados Unidos, por lo que un alto número de pobladores estadounidenses acude a territorio mexicano para conseguirlos. ${ }^{3}$ Este hecho ha generado que en ciudades como Tijuana exista una visible acumulación de farmacias y consultorios médicos muy cercanos a la línea internacional, o bien, en las avenidas más transitadas por visitantes estadounidenses, donde se promocionan medicamentos controlados con distintas ofertas y precios rebajados. Este tipo de contrastes, junto a muchos otros, son algunos de los que se experimentan en la convivencia de la frontera Tijuana-San Diego.

\section{Tráfico de armas en la frontera}

Las armas y la violencia asociada a ellas son parte integral del imaginario social de los mexicanos, particularmente entre aquellos que viven en los estados fronterizos y otras áreas controladas por los carteles de la droga. A pesar de ello, el uso y compraventa de armas ha estado fuertemente regulado tanto por las autoridades mexicanas como por las autoridades fronterizas estadounidenses. Esto contrasta con la facilidad con la que se pueden adquirir armas de fuego en Estados Unidos, en donde incluso pueden comprarse en tiendas de autoservicio como Walmart. Esta situación genera la existencia de advertencias para quienes cruzan en dirección a México (Figura 2). Aunque los ciudadanos mexicanos tienen el derecho a poseer armas de fuego para su propia seguridad, la adquisición de estas solo puede ocurrir a través de la tienda de la Secretaría de la Defensa Nacional ubicada en la Ciudad de México, luego de haber aprobado un examen psicológico y demostrar la necesidad de poseer un arma de fuego. Una vez superadas estas barreras, la selección de calibres y modelos permitidos

\footnotetext{
${ }^{3}$ Algunos de estos medicamentos son somacid (carisoprodol), vicodin (hydrocodone /paracetamol), xanax (alprazolam), valium (diazepam), rivotril (clonazepam).
} 
es sumamente limitada, aunque las posibilidades aumentan si la persona es miembro de un club de tiro deportivo, o bien, diplomático, servidor público o celebridad (Ley Federal de Armas de Fuego y Explosivos, 1972).

Figura 2. Señalamiento en el freeway 1-5 de San Ysidro, en dirección hacia México

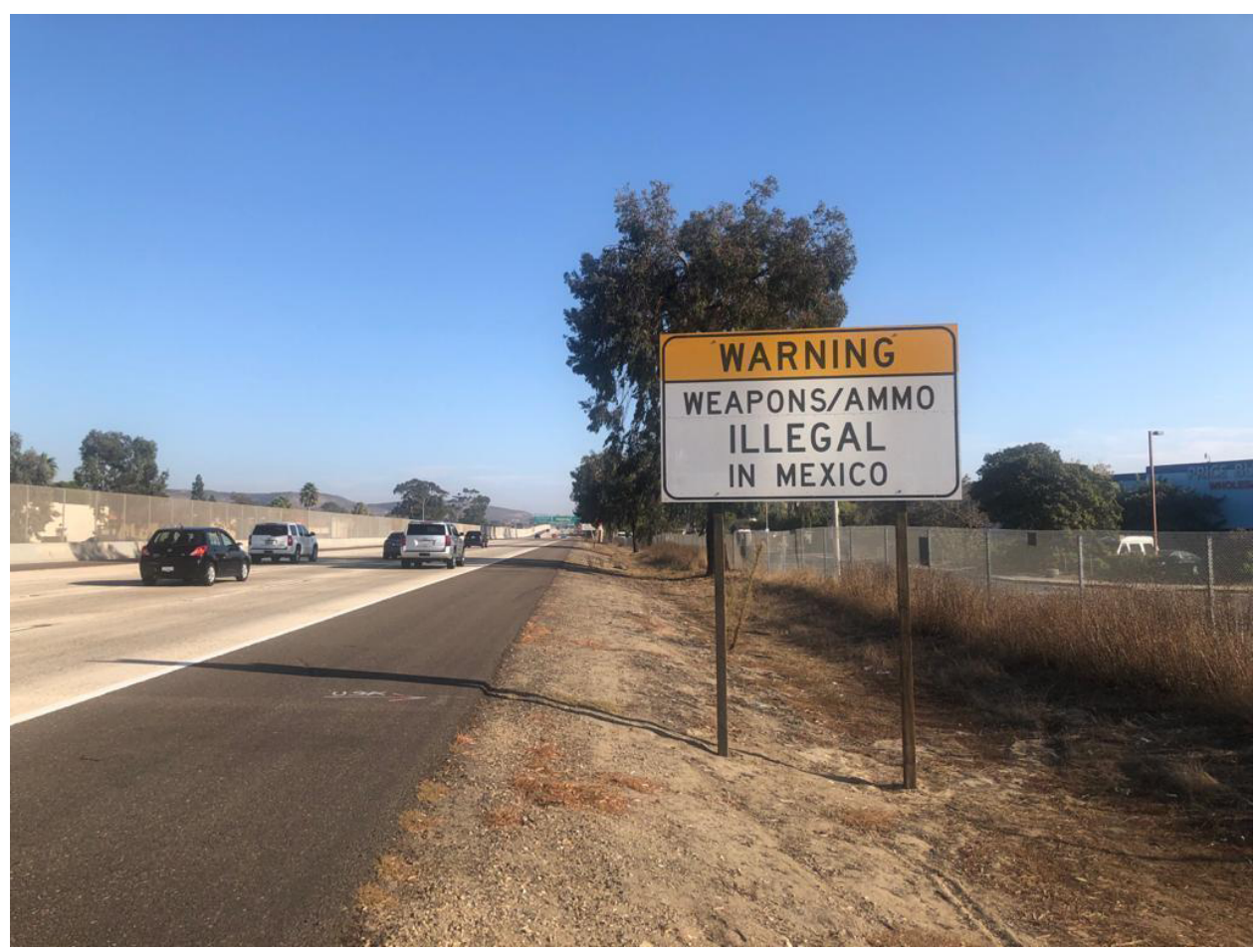

Foto: Alberto Hernández, 2019

Es importante mencionar que, a pesar de dichas restricciones, el tráfico ilegal permite que todo tipo de armas circulen y sean accesibles dentro del territorio mexicano, ya sean pistolas, rifles, ametralladoras e incluso rifles de francotirador de grado militar; la presencia y el incremento de este tipo de armas se dio a partir de la guerra contra las drogas que inició el presidente Felipe Calderón en 2006 (Shirk, 2011). Al tomar en cuenta este contexto, la mayoría del tráfico de armas ocurre en un sentido norte-sur y suele ser perpetrado por personas sin antecedentes penales, particularmente las esposas y parejas de personas pertenecientes a un grupo delictivo. Otra modalidad de tráfico es mediante la contratación de personas sin antecedentes penales para realizar la transacción. De acuerdo con la Organización de las Naciones Unidas (ONU), un aproximado de 20000 armas son introducidas a México de manera ilegal cada año, sobre todo a través de Texas, California y Arizona, con un destino final enfocado a los estados controlados por los carteles de droga más grandes del país: Michoacán, Tamaulipas, Sinaloa, Sonora, Baja California y Chihuahua (United Nations Office on Drugs and Crime [UNODC], 2010). En este sentido, el "tráfico hormiga" compone la mayoría de los incidentes relacionados con la compra-venta de armas. Según el reporte unODC, existen 6700 vendedores autorizados de armas de fuego en 
la región fronteriza, que representan 12\% del total de 55000 vendedores autorizados en Estados Unidos. El mismo reporte indica que $70 \%$ de las armas confiscadas en México provienen del vecino país, principalmente de Texas (39\%), California (20\%) y Arizona (10\%). Estos datos fueron actualizados en 2014 por la Oficina de Fiscalización Superior del Gobierno de los Estados Unidos (GAO, por sus siglas en inglés), como lo muestra la Figura 3.

Independientemente de su origen, la mayoría de estas armas pasan a territorio mexicano a través de Tamaulipas, de acuerdo con el número de armas confiscadas en dicho estado, y, aunque se ha establecido que la mayoría de las armas tienen un origen legal, también existe contrabando de otro tipo de armas que ingresan a través de la frontera guatemalteca (Latin American Herald Tribune, 2011), que suelen ser adquiridas por los grupos delictivos luego de los choques violentos entre ellos y las fuerzas armadas y la policía. En el peor de los casos, estas armas son producto de asaltos directos a los arsenales y a las estaciones militares y policiacas por grupos delictivos (Borderland Beat, 2010).

Figura 3. Origen de las armas de contrabando en México

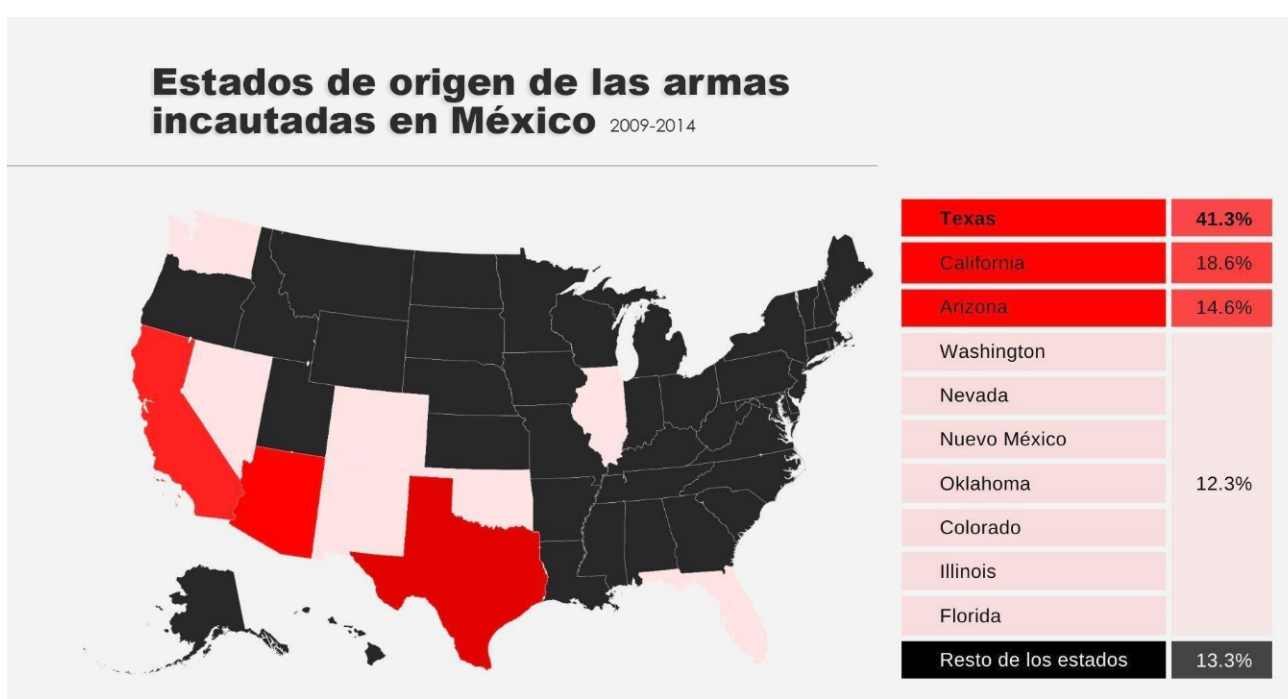

Fuente: Oficina de Fiscalización Superior del Gobierno de Estados Unidos (GAO). Elaborado por Melissa Urquidez

Las armas más comunes que son traficadas son los rifles tipo AR y AK y pistolas FN. La mayoría de las variantes de AK provienen de Centroamérica, Sudamérica, el Medio Oriente, África y el Sureste Asiático. La mayoría de las variantes AR provienen de Estados Unidos, Vietnam y países de la otan como España, además de otros como Corea del Sur y Colombia (Goodman \& Marizco, 2010). En ocasiones, los grupos delictivos logran contrabandear rifles de alto poder de uso exclusivo militar, como el Barrett calibre .50 (Vicenteño, 2020). Es importante mencionar que, a pesar de los esfuerzos para combatir el tráfico de armas, operativos como Gunrunner, Fast and Furious, Too Hot to Handle, Wide Receiver y White Gun, destinados a dibujar las 
trayectorias de tráfico e identificar a los traficantes, han sido contraproducentes, al incrementar el volumen de armas en circulación en México. Debido a esto, este tipo de operativos dejaron de funcionar en 2011 (Pavlich, 2012).

\section{Túneles transfronterizos}

En 2008 se descubrió un túnel entre Tijuana y San Ysidro, a escasos metros de la garita del cruce fronterizo de San Ysidro. Este evento sorprendió a las autoridades en ambos lados de la frontera. Del lado mexicano, el túnel estaba escondido en el interior de una casa de dos pisos, construida durante la década de 1950. El pasaje contaba con iluminación y ventilación, y se estima que funcionó durante casi 30 años. Su descubrimiento fue fortuito, gracias a los trabajos de remodelación que se llevaban a cabo del lado estadounidense (Ramírez, 2020). Aunque este es uno de los casos más famosos dada su cercanía con la garita, otros narcotúneles han sido descubiertos con el paso de los años: para 2016, las autoridades habían descubierto 181 túneles y en 2020 se descubrió el túnel más largo hasta la fecha, en una de las zonas industriales de Tijuana, la Mesa de Otay (Fry, 2020). Otros túneles que han sido descubiertos en los últimos años son el túnel acuático entre Mexicali y Caléxico en 2015 y el túnel de 2020 entre Brownsville y Matamoros por debajo del Río Bravo (Marty, 2015; EFE, 2020). Aunque la mayoría de estos túneles se ubican en dirección de sur a norte y son utilizados para el tráfico de sustancias ilícitas, estos pasajes también son usados para el tráfico de personas a través de la frontera.

\section{Otras estrategias de contrabando: trenes, camiones y furgonetas}

Cuando se firmó el Tratado de Libre Comercio para América del Norte (TLCAN) de 1994, el número de productos que se comercializaban entre México, Canadá y Estados Unidos se incrementó de manera exponencial. Hasta 2020, con excepción de los puertos marítimos, la totalidad de los productos de exportación e importación atravesaba por alguna de las 55 garitas terrestres. De acuerdo con la BTs, en 2019 ocurrieron más de 10000000 de cruces comerciales a través de camiones, furgonetas y trenes (BTs, s. f.).

Se debe considerar que $79 \%$ de los cruces comerciales se concentran en cinco ciudades: Nuevo Laredo, Tijuana, Juárez, Reynosa y Nogales (Tabla 1). Sobre los objetos que cruzan, la mayoría consiste en autopartes, vehículos, materiales de manufactura, vegetales y comida. Dado el volumen de vehículos, es común que deban esperar hasta 24 horas para poder cruzar la frontera. A pesar de la tecnología de punta en cada una de las garitas, los contrabandistas siempre encuentran oportunidades para llevar a cabo operaciones de menor y mediana escala, ya sea combinando los productos ilegales (cocaína, cristal, heroína, marihuana, entre otros) con la mercancía permitida, o bien, ocultándolos en compartimentos secretos dentro de los mismos vehículos, desde furgonetas hasta camiones (Tabla 2). 
Tabla 1. Cruces comerciales entre México y Estados Unidos, 2019

\begin{tabular}{|c|c|}
\hline Garita & Número de cruces \\
\hline Nuevo Laredo, Tamps.-Laredo, TX & 3741876 \\
\hline Tijuana (Otay), B.C.-Otay Mesa, CA & 1570408 \\
\hline Cd. Juárez, Chih.-El Paso, TX & 1340083 \\
\hline Reynosa, Tamps.-Hidalgo, TX & 1068697 \\
\hline Nogales, Son.-Nogales, AZ & 584782 \\
\hline Mexicali, B.C.-Caléxico East, CA & 558722 \\
\hline Piedras Negas, Coah.- Eagle Pass, TX & 464067 \\
\hline Matamoros, Tamps.-Brownsville, TX & 418269 \\
\hline San Jerónimo, Chih.-Santa Teresa, NM & 248628 \\
\hline Cd. Acuña, Coah.-Del Rio, TX & 113970 \\
\hline Tecate, B.C.-San Diego, CA & 88114 \\
\hline Nuevo Progreso, Tamps.-Progreso Lakes, TX & 76103 \\
\hline Camargo, Tamps.-Rio Grande City, TX & 65066 \\
\hline San Luis Río Colorado, Son.-San Luis, AZ & 61735 \\
\hline Agua Prieta, Son.-Douglas, AZ & 42334 \\
\hline Puerto Palomas, Chih.-Columbus, NM & 30446 \\
\hline Ojinaga, Chih.-Presidio, TX & 18616 \\
\hline Cd. Miguel Alemán, Tamps.-Roma, TX & 14060 \\
\hline Naco, Son.-Naco, AZ & 5166 \\
\hline Sonoita, Son.-Lukeville, AZ & 618 \\
\hline Número total de cruces & 10511760 \\
\hline
\end{tabular}

Fuente: BTS, s. f. 
Tabla 2. Cruces comerciales según tipo de transporte, 2019

\begin{tabular}{|l|r|}
\hline \multicolumn{1}{c|}{ Tipo de transporte } & Número de cruces \\
\hline Furgonetas & 5955884 \\
\hline Camiones & 4093132 \\
\hline Contenedores & 451071 \\
\hline Trenes & 11673 \\
\hline Número total de cruces & $\mathbf{1 0 5 1 1 7 6 0}$ \\
\hline
\end{tabular}

Fuente: BTs, s. f.

Desde hace al menos cinco años, en distintos medios de comunicación de México y el mundo se han visibilizado las nuevas estrategias de traficantes para cruzar sustancias ilícitas por las fronteras, mediante el uso de pepinos y aguacates de plástico, o bien, cargamentos de papayas, entre otras frutas, y verduras de mayor tamaño que sirven de camuflaje a este tipo de cargamentos. Tan solo el 24 de febrero de 2021, la свP incautó más de 5500 kilogramos de marihuana dentro de un cargamento de papaya en el puerto fronterizo de Mesa de Otay en Tijuana. Este cargamento tendría un valor de alrededor de 27000000 de dólares, según informaron autoridades estadounidenses (EFE, 2021).

\section{Conclusiones}

Los anteriores ejemplos permiten ilustrar la distinta condición y estatus legal que adquieren mercancías y productos según el lado de la frontera donde se ubiquen, hecho que reconfigura en la práctica las comprensiones y efectos que tiene lo legal y lo ilegal en una zona fronteriza como la de México con Estados Unidos.

Lo referido por Osório Machado (2000) en torno a la redefinición de límite y territorio fronterizo a partir de las prácticas de legalidad e ilegalidad se torna visible en el caso de la frontera Tijuana-San Diego. También, el estudio de Osório Machado y colaboradores (2009), respecto a las formas de interacción y relación económica en territorios de frontera, puede ser traído al caso de la frontera de México con Estados Unidos, ya que, si bien hay un límite jurídico y una actuación institucional en ambos países, los sentidos de tiempo y espacio que se producen en zonas fronterizas generan dinámicas de interacción ajenas a las normas establecidas por el poder estatal, tal y como ocurre con el contrabando y otras actividades ilegales en los puertos internacionales.

En otras palabras, las personas que forman parte de estos circuitos del comercio informal entre Estados Unidos y México, y particularmente en el caso de la región fronteriza de Tijuana y San Diego, aprovechan las necesidades de las poblaciones locales, la lejanía del poder político administrativo central, así como el desconocimiento u omisión de los gobiernos estatales, para continuar con el contrabando desde diferentes modalidades y esquemas de cruce. Por esta razón, las prácticas sociales económicas en fronteras, sean legales o ilegales, parecieran articularse al margen de disposiciones estatales, y revelan otro tipo de integración económica a través de los mercados informales y los circuitos de la ilegalidad, donde se aprovecha la condición porosa de las zonas de frontera. 
Lo anterior, sumado a la actuación dispar de los Estados en la administración y control de sus fronteras, particularmente en los mercados de la ilegalidad, revelan de qué manera se construye lo que ha sido nombrado por Gustavo Lins Ribeiro como una "globalización desde abajo", término con el que se refiere a las economías informales que ocurren en sincronía con las economías formales, es decir, las globalizaciones "desde arriba". Esta noción sigue siendo adecuada para representar cómo los mercados de la ilegalidad siguen transformando las economías regionales fronterizas (Lins Ribeiro, 2006). La existencia de estos mercados devela aún más el problema de la distribución desigual de poder y expone, además, de manera explícita, la relación simbiótica entre los gobiernos y las prácticas ilegales. Estas características permiten ver que las relaciones entre lo legal y lo ilegal son "multifacéticas y complejas, e involucran intereses normativos, políticos y morales diversos" (Lins Ribeiro, 2018, p. 86).

Sin duda, los nuevos estudios que se hagan sobre este fenómeno deberán tomar en cuenta los cambios en las dinámicas de flujos transfronterizos a raíz de las restricciones impuestas por las medidas sanitarias para combatir la pandemia del COVID-19, situación que afectó no solo al contrabando y al tráfico hormiga, sino también a las importaciones y exportaciones de productos en general. A partir del 21 de marzo de 2020, el gobierno federal de Estados Unidos decretó un cierre parcial de las fronteras terrestres con México y Canadá, medida que se prolongó mes tras mes hasta alcanzar más de un año de vigencia. A pesar de este cierre parcial, el flujo transfronterizo no se redujo por completo, sino que se hizo más lento a raíz de las revisiones sanitarias y las restricciones de cruce destinadas a permitir únicamente el paso a ciudadanos y residentes estadounidenses y trabajadores esenciales de origen extranjero.

El acceso a México, por el contrario, permaneció abierto para peatones y vehículos, aunque se establecieron medidas básicas sanitarias y se restringieron los trámites para importación de mercancías. Las consecuencias de estas medidas se vieron reflejadas en la vida cotidiana de las personas en comunidades transfronterizas, las cuales se vieron ante situaciones de escasez de productos disponibles únicamente en Estados Unidos, desde dulces y alimentos hasta ropa, calzado, electrodomésticos, muebles y productos electrónicos. Asimismo, una serie de negocios formales e informales se vieron orillados a cerrar tanto por falta de mercancía, como por el flujo reducido de clientes. Este periodo de crisis sanitaria, sin embargo, también representó una oportunidad para el surgimiento de nuevos actores y dinámicas de cruce de productos legales y de contrabando, como el encargo de dichos productos a personas con capacidad de cruce mediante redes sociales y plataformas digitales, así como el aumento de precios por la prestación de este servicio en función de distancia, cantidad y volumen.

Finalmente, es importante resaltar cómo debido al aumento de las dificultades para el contrabando a causa de la pandemia, se impactó severamente a comerciantes de ambos lados de la frontera, cuyos efectos fueron más visibles en los comercios de San Ysidro, California, y de Tijuana. Condición que llevó a muchos comerciantes al cierre de negocios, o bien, a la suspensión o reducción de sus actividades a causa de la falta de mercancía y clientes. Estos hechos demuestran el rol tan importante que ocupan este tipo de actividades para miles de personas en el espacio fronterizo, y que los ha llevado a ingeniar nuevas estrategias y modos de operación que les permitan seguir sorteando estos obstáculos para el cruce de productos y mercancías por la frontera.

Si bien el impacto del contrabando es diferenciado en términos de los productos y mercancías que cruzan, ya sea en dirección norte-sur o sur-norte, es innegable que 
dentro de este gran mercado también se cobija el tráfico de drogas, armas y personas. Por lo que aun cuando este tipo de prácticas entre lo legal y lo ilegal constituye oportunidades económicas para grandes segmentos de las poblaciones fronterizas, también representa grandes costos sociales, además de acentuar problemáticas como la venta y consumo de drogas, el aumento de adicciones a drogas sintéticas, así como mayores hechos de violencia en ambos lados de la frontera. Por lo que se torna necesario continuar con el estudio de estas prácticas entre lo legal y lo ilegal y la manera en que se articulan e impactan en el espacio transfronterizo.

\section{Referencias}

Anderson, J. \& O’Dowd, L. (1999). Borders, border regions and territoriality: contradictory meanings, changing significance. Regional Studies, 33(7), 593-604. https://www.tandfonline.com/doi/abs/10.1080/00343409950078648

Borderland Beat. (2010, 28 de septiembre). State Police arsenal raided in Chihuahua City. http://www.borderlandbeat.com/2010/09/state-police-arsenal-raided-in.html

Brigham, A. P. (1919, abril). Principles in the determination of boundaries. The Geographical Review, 7(4), 201-219. https://www.loc.gov/resource/dcmsiabooks. principlesindete00brig/?sp=5\&r=0.062,0.323,0.942,0.805,0

Bureau of Transportation Statistics (BTS). (s. f.). Border crossing/entry data. https://www. bts.gov/content/border-crossingentry-data

Bureau of Transportation Statistics (втs). (2019). Border Crossing Entry Data. https:// explore.dot.gov/views/BorderCrossingData/Monthly?:isGuestRedirectFromVizportal=y\&:embed $=y$

Dilla Alfonso, H. \& Neira Orjuela, F. (Eds.). (2020). Donde el pedernal choca con el acero. Hacia una teoría crítica de las fronteras latinoamericanas. RIL editores/Universidad Arturo Prat. https://www.academia.edu/49015997/Donde_el_pedernal_choca_con_el_acero_hacia_una_teor\%C3\%ADa_cr\%C3\%ADtica_de_las_fronteras_latinoamericanas

Donnan, H. \& Wilson, T. (2001). Borders: frontiers of identity, nation and state. Berg.

Dorfman, A. (2020). Geografía moral del contrabando. En H. Dilla Alfonso \& F Neira Orjuela (Eds.), Donde el pedernal choca con el acero. Hacia una teoría crítica de las fronteras latinoamericanas (pp. 155-174). RIL editores/Universidad Arturo Prat. https://www.academia.edu/49015997/Donde_el_pedernal_choca_con_ el_acero_hacia_una_teor\%C3\%ADa_cr\%C3\%ADtica_de_las_fronteras_latinoamericanas

EFE. (2020, 26 de agosto). Encuentran túnel entre México y Eu construido bajo el Río Bravo. El Universal. https://www.eluniversal.com.mx/mundo/encuentran-tunel-entre-mexico-y-eu-construido-bajo-el-rio-bravo

EFE. (2021, 24 de febrero). Incautan marihuana valorada en 27 millones en un cargamento de papayas. https://www.efe.com/efe/america/mexico/incautan-marihuana-valorada-en-27-millones-un-cargamento-de-papayas/50000545-4473367

Fry, W. (2020, 29 de enero). Longest cross-border drug tunnel in history discovered in Otay Mesa. The San Diego Union Tribune. https://www.sandiegouniontribune. 
com/news/border-baja-california/story/2020-01-29/longest-cross-border-drugtunnel-in-history-discovered-in-otay-mesa

Fuentes, C. (2017). Las fronteras de México: nodos del sistema global de las drogas prohibidas. Flacso-IDRC-CRDI-El Colef.

Gauthier, M. (2009). The fayuca hormiga of used clothing and the fabric of the Mexico-us border [Tesis doctoral, Concordia University]. https://spectrum.library.concordia. ca/976420/1/NR63375.pdf

Goodman, C. \& Marizco, M. (2010). us firearms trafficking to Mexico: new data and insights illuminate key trends and challenges. Woodrow Wilson International Center for Scholars-Mexico Institute/Trans-Border Institute-University of San Diego. https://www.cwagweb.org/wp-content/uploads/2016/08/U.S.-Firearms-Trafficking-to-Mexico-Goodman-Final.pdf

Grimson, A. (2011). Los límites de la cultura. Críticas de las teorías de la identidad. Siglo XXI.

Hartshorne, R. (1936). Suggestions on the terminology of political boundaries. Annals of the Association of American Geographers, 26(1), 56-57.

Hernández, A. (2018). Prácticas culturales y religiosas en contextos de cambio y movilidad. En N. Sanz \& J. Valenzuela (Coords.), Cultura, migración y desarrollo. Visión y acción desde México (pp. 121-134). El Colegio de la Frontera Norte/unesco.

Latin American Herald Tribune. (2011, 31 de marzo). Mexican cartels get heavy weapons from Central America, U.s. cables say. http://www.laht.com/article.asp?CategoryId $=14091 \&$ ArticleId $=390473$

Lins Ribeiro, G. (2006). Economic globalization from below. Etnográfica, 10(2), 233249. https://repositorio.unb.br/bitstream/10482/17600/1/ARTIGO_EconomicGlobalizationBelow.pdf

Lins Ribeiro, G. (2018). Otras globalizaciones. UAM/Gedisa.

Loya, A. M., González-Stuart, A. \& Rivera,J. O. (2009). Prevalence of polypharmacy, polyherbacy, nutritional supplement use and potential product interactions among older adults living on the United States-Mexico border. Drugs $\mathcal{E}$ aging, 26(5), 423436. https://link.springer.com/article/10.2165/00002512-200926050-00006

Marty, B. (2015, 20 de agosto). Encuentran narcotúnel subacúatico en frontera entre México y EE. UU. Panam Post. Noticias y Análisis de las américas. https://es.panampost. com/belen-marty/2015/08/20/encuentran-narcotunel-subacuatico-en-frontera-entre-mexico-y-ee-uu/

Newman, D. (2006). Borders and bordering. Toward an interdisciplinary dialogue. European Journal of Social Theory, 9(2), 171-186. https://www.researchgate.net/ publication/249710394_Borders_and_BorderingTowards_an_Interdisciplinary_Dialogue

Osório Machado, L. (2000). Límites e fronteras: Da alta diplomacia aos circuitos da ilegalidade. Territorio, 5(8), 7-23.

Osório Machado, L., Reyes, A. \& Monteiro, L. (2009). Building walls, breaking barriers: territory, integration and the rule of law in frontier zones. Journal of Borderland Studies, 24(3), 97-114. https://www.tandfonline.com/doi/abs/10.1080/0886565 5.2009 .9695742

Pavlich, K. (2012). Fast and furious: Barack Obama's bloodiest scandal and the shameless cover-up. Regnery Publishing. 
Piñera, D. (1985). Historia de Tijuana: Semblanza general. Centro de Investigaciones Históricas, UNAM-UABC.

Prieto, R. (2016, 29 de enero). Los trece milloes de la frontera. Animal Político. https:// www.animalpolitico.com/columna-invitada/los-trece-millones-de-la-frontera/

Ramírez, C. (2020, 23 de febrero). La Casa del Túnel: el narcotúnel que es más viejo que El Chapo y ya puedes visitar. Reporte Índigo. https://www.reporteindigo.com/piensa/la-casa-del-tunel-el-narcotunel-que-es-mas-viejo-que-el-chapo-y-ya-puedesvisitar/

Sandoval, E. (2012). Infraestructuras transfronterizas. Etnografía de itinerarios en el espacio social Monterrey-San Antonio (Publicaciones de la Casa Chata). Colef/ciesas.

Sandoval, E. (2018). Pacas de a libra. Control territorial del comercio de ropa usada en la frontera de Texas y más acá. En E. Sandoval (Coord.), Violentar la vida en el norte de México. Estado, tráficos y migraciones en la frontera con Texas (pp. 327-360). CIESAS/Plaza y Valdés.

Senado de la República/Centro de Estudios Internacionales Gilberto Bosques. (2017, 1 de junio). Panorama actual de la frontera entre México y Estados Unidos (nota informativa). https://centrogilbertobosques.senado.gob.mx/docs/NI_FronteraMX_EEUU_010617.pdf

Shirk, D. A. (2011). Transnational crime, us border security, and the war on drugs in Mexico. Trans-Border Institute-University of San Diego. http://catcher.sandiego.edu/ items/peacestudies/110331-Shirk-Testimony.pdf

United Nations Office on Drugs and Crime (UNODC). (2010). The globalization of crime. A transnational organized crime threat assessment. United Nations publication. https://www.unodc.org/documents/data-and-analysis/tocta/TOCTA_Report_2010_low_res.pdf

Vicenteño, D. (2020, 31 de agosto). Mujer intenta entrar a México con un fusil Barrett y ocho mil cartuchos. Excelsior. https://www.excelsior.com.mx/nacional/mujerintenta-entrar-a-mexico-con-un-fusil-barrett-y-ocho-mil-cartuchos/1403034

Vinasco, J. (2018). We can(nabis) in Tijuana. Un análisis de la incidencia de la regulación del cannabis en California sobre las prácticas de consumo transfronterizo de residentes en Tijuana" [Tesis de doctorado, El Colegio de la Frontera Norte]. https://www. colef.mx/posgrado/tesis/20141118/

Alberto Hernández Hernández

Mexicano. Doctor en sociología por la Universidad Complutense de Madrid. Profesorinvestigador del Departamento de Estudios en Administración Pública de El Colegio de la Frontera Norte (México); actualmente es presidente de la misma institución. Líneas de investigación: migración, fronteras y estudios culturales. Publicación reciente: Hernández Hernández, A. (Coord.). (2020). Puentes que unen y muros que separan. Fronterización, securitización y procesos de cambio en las fronteras de México y Brasil. El Colef/Universidade Federal de Mato Grosso do Sul. 